DOI: $10.17516 / 2782-2214-0035$

УДК 338.46

\title{
DEVELOPMENT OF A METHOD FOR ANALYSIS OF PROCESSES OCCURRING DURING THE INTERACTION OF THE ENTERPRISE WITH THE EXTERNAL ENVIRONMENT IN THE PRODUCT SALES PROCESS
}

\author{
Alexander V. Tsatsorin* \\ Siberian Federal University, Krasnoyarsk, Russian Federation
}

\begin{abstract}
The article analyzes enterprises that sell products through various channels. The development of the method is based on the use of graphs. The sources of the data were the observation of several companies that sell their products using different channels.

As a result of the study, it was found that this method can presumably be used in modeling the interaction of enterprises with the external environment both at the stage of creating a business and at the stage of its functioning.

The study did not assess the financial component of specific enterprises, the number of employees, time of presence on the market, marketing strategy and other parameters. The assessment was carried out solely by the presence of a connection between the enterprise and the subject of the external environment and their number.
\end{abstract}

Key words: networking, graphs, matrices, business modeling, business processes.

Citation: Tsatsorin, A. V. (2021). Development of a method for analysis of processes occurring during the interaction of the enterprise with the external environment in the product sales process. Trade, service, food industry. Vol. 1(4). Pp. 333-344.

\section{РАЗРАБОТКА МЕТОДА АНАЛИЗА ВЗАИМОДЕЙСТВИЙ ПРЕДПРИЯТИЯ С ВНЕШНЕЙ СРЕДОЙ В ПРОЦЕССЕ СБЫТА ПРОДУКЦИИ} Александр Владиславович Цацорин ${ }^{*}$ ФГАОУ ВО «Сибирский федеральный университет», Красноярск, Российская Федерация

Аннотация. Проанализированы предприятия, которые занимаются сбытом продукции, с помощью метода, основанного на использовании графов. Источниками данных послужило наблюдение за несколькими компаниями, которые реализуют свою продукцию, используя различные каналы.

Предположительно данный метод может быть использован в моделировании взаимодействия предприятий с внешней средой на этапе как создания бизнеса, так и его функционирования.

В процессе исследования не оценивалась финансовая составляющая конкретных предприятий, численность работников, время присутствия на рынке, маркетинговая стратегия и другие параметры. Оценка происходила исключительно по наличию связи между предприятием и субъектами внешней среды и их количеству.

Ключевые слова: сетевое взаимодействие, графы, матрицы, бизнесмоделирование, бизнес-процессы.

Введение. Развитие бизнеса в настоящее время идет высокими темпами. Большое значение в данном процессе имеет способность предприятий кооперироваться и достигать совместных целей используя ресурсы и возможности партнеров. Методы

(C) Siberian Federal University. All rights reserved

* Corresponding author E-mail address: tsatsorin@mail.ru 
оценки таких способов кооперации требуют улучшения для того, чтобы компании имели возможность анализировать, планировать и прогнозировать свою деятельность.

Цель данного исследования - разработка метода анализа взаимодействия предприятия с внешней средой.

Среди задач здесь стоит выделить такие:

- описание процессов, которые автор считает необходимым рассмотреть в данной работе;

- описание субъектов внешней среды исследуемых предприятий;

- попытка применения графов для анализа моделей взаимодействия;

- подготовка выводов о результатах применения графов при анализе моделей взаимодействия.

Материалы и методы. В ходе исследования использованы следующие методы: наблюдение, сбор информации, анализ информации, моделирование.

Количество исследуемых предприятий (филиалов) - 4.

В качестве основы для анализа эффективности служат графы, т. к. с их помощью можно достаточно наглядно показать как сами субъекты взаимодействия, так и процессы, происходящие между ними.

Обсуждение. Исследованию сетевого взаимодействия, а также методам оценки эффективности посвящено множество работ как отечественных, так и зарубежных экономистов. Так, Н. В. Рубцовой осуществлен критический обзор методик, посвященных оценке эффектов взаимодействия субъектов в социально-экономических системах. На 2020 г. исследователь выделяет шесть вариантов, используемых другими авторами, и в результате предлагает собственную методику.

Стоит отметить, что все рассмотренные методики основаны на некоем «синергетическом» эффекте.

В рамках настоящей статьи автор предлагает использовать иной метод оценки и анализа взаимодействия субъектов экономических отношений, основанный не на синергетическом эффекте, а исключительно на количественных показателях различных процессов, которые происходят между рассматриваемым предприятием и его внешней средой.

Для целей визуального представления процессов взаимодействия предприятия с внешней средой используем графы как достаточно наглядный способ показать структуру самих процессов и их направленность. Теория графов - это область дискретной математики, особенностью которой является геометрический подход к изучению объектов и связей между ними, причем сами объекты будут показаны как вершины графа, а процессы, происходящие между ними, как ребра. Направленность процесса показана стрелкой на ребре. Некоторые взаимодействия внутри объекта представлены как петлевой граф (имеющий ребро, начало и конец которого находятся в одной и той же вершине). Подсчет количества ребер (процессов) среди вершин (объектов взаимодействий) потенциально может помочь в анализе и оценке внешней среды предприятия.

Модель. Осуществлено наблюдение за несколькими организациями, которые занимаются сбытом продукции, и их взаимодействием с внешней средой. Определены модели сотрудничества предприятий в процессе поставки товара от поставщика до конечного потребителя. Разумеется, данную методику предположительно можно использовать в процессе анализа взаимодействия участников рынка в различных процессах, в т.ч. производстве, оказании услуг и прочее.

В данной работе представлены три модели взаимодействия предприятий в процессе поставки товара, которые автор называет «классической», «сетевой», «платформенной». В каждой из этих моделей присутствуют различные участники 
рынка и, соответственно, процессы, происходящие между ними: хранение и учет, упаковка, физическое перемещение, выкладка товара, информирование о товаре (маркетинговые мероприятия, реклама и т.д.), проведение финансовых расчетов. Само производство товара и его появление у поставщика автор не рассматривает с целью экономии времени.

Далее приведено описание тех процессов, которые были выявлены в ходе наблюдения.

Процесс хранения и учета товара представляет собой ряд операций, необходимых для сохранения потребительских свойств товара до поставки конечному потребителю, его документационного сопровождения.

Упаковка служит выполнению различных целей, таких как привлекательный внешний вид для конечного потребителя, сохранение потребительских свойств товара в процессе его физического перемещения.

Физическое перемещение товара представляет собой процесс перевозки товара между объектами взаимодействия.

Выкладка товара является способом визуального представления товара для посредника или конечного потребителя с целью появления у последнего желания его приобрести.

Информирование о товаре включает в себя весь комплекс мероприятий, необходимый для доведения информации о существовании товара, его потребительских свойств до участников процесса поставки. Включает в себя маркетинговую стратегию, рекламные мероприятия, и т. д.

Финансовые расчеты представляют собой совокупность операций движения денежных средств между участниками процесса поставки товара.

В процессе исследований определены участники процесса, (разумеется, данный список не является исчерпывающим; при анализе других предприятий он может отличаться):

- поставщик товара (исследуемое предприятие) - производитель или продавец товара, который рассматривается в рамках конкретной модели (начальное звено в процессе взаимодействия);

- партнер по упаковке - предприятие, предоставляющее мощности для упаковки товара поставщика;

- партнер по складированию и учету - предприятие, предоставляющее мощности для складирования товара и его учета;

- транспортный партнер - предприятие, предоставляющее мощности для физического перемещения товара между участниками процесса поставки;

- информационный партнер - предприятие, предоставляющее мощности для создания маркетинговых мероприятий, рекламы и т. д.;

- оптовая компания - участник процесса поставки товара, осуществляющий закупку товаров у поставщиков и их реализацию в розничные сети;

- розничная сеть - участник процесса поставки товара, осуществляющий непосредственную передачу товара конечному потребителю;

- платформа - участник процесса поставки товара, осуществляющий предоставление информации о наличии товара для большого числа потенциальных потребителей и взаимодействие с поставщиком товара.

Следует отметить, что участники процесса поставки товара - это множество компаний, которые могут быть задействованы в нем. Например, оптовая компания, изображенная в модели, есть множество оптовых компаний, с которыми взаимодействует исследуемое предприятие (поставщик). 
Рассмотрим классическую модель взаимодействия (рис. 1), в которой автор выделяет следующих участников процесса поставки товара: поставщик, оптовая компания, партнер по реализации, потребитель. Здесь сбыт продукции осуществляется поставщиком с помощью оптовых компаний, которые, в свою очередь, поставляют продукцию розничным сетям, где уже происходит доведение товара до конечного потребителя.

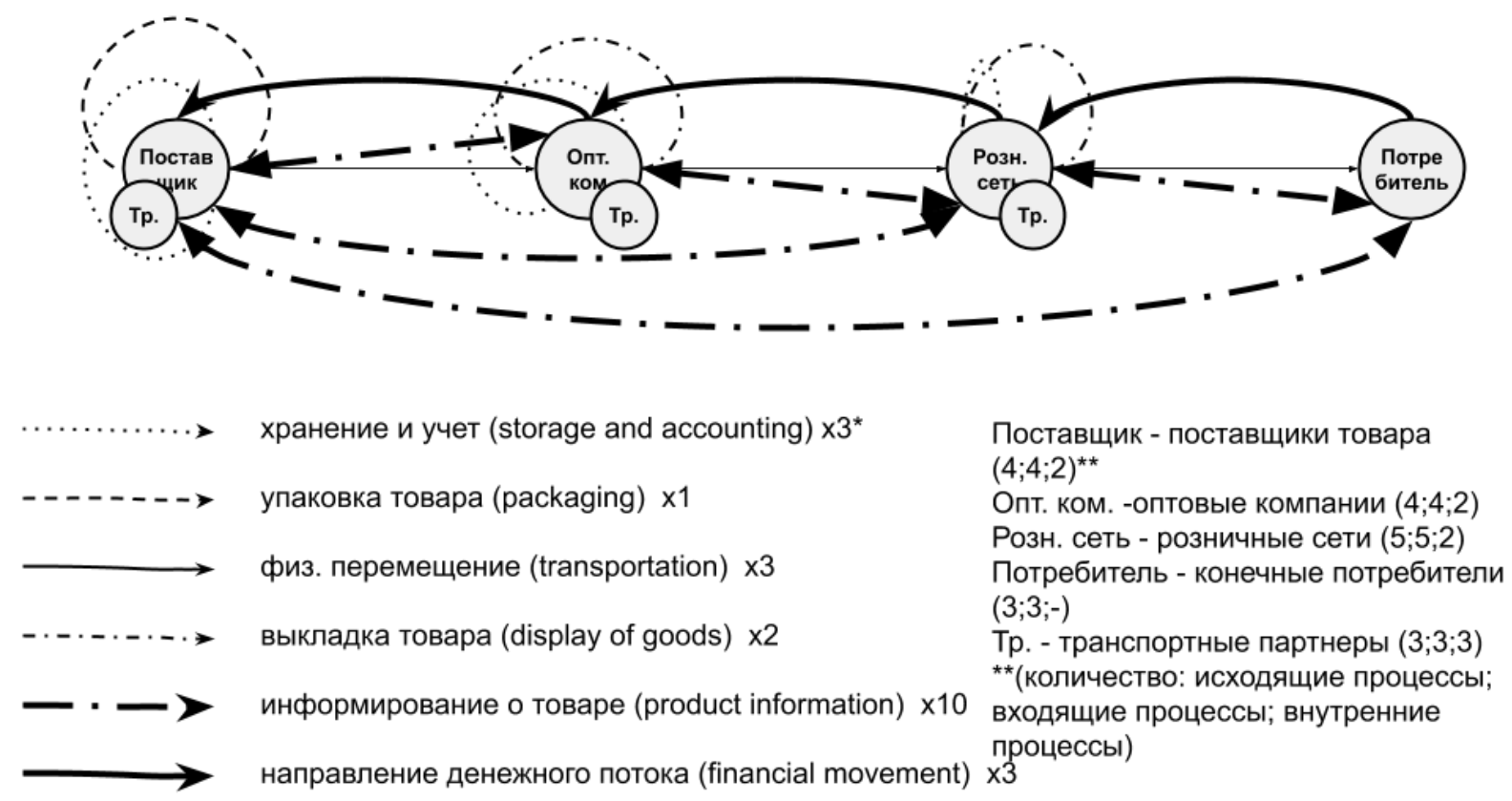

* xn = количество процессов на графике

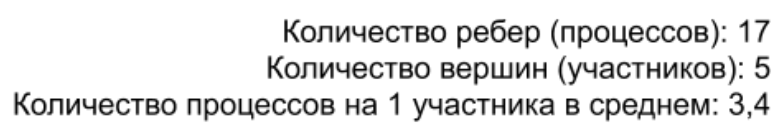

Рисунок 1. Схема классической модели взаимодействия

Figure 1. Scheme of the classical interaction model

Данный рисунок представляет собой граф, где участники процесса обозначены как вершины, а происходящие между ними процессы представлены как ребра. Ориентированные ребра показывают, что у процесса есть выход, находящийся в одной вершине и вход, находящийся в другой вершине. Неориентированные ребра (информирование о товаре) подразумевают процесс, не имеющий строгого выхода и входа, так как постоянно происходит обмен информацией, обратная связь и т.д. Петли показаны как процессы, происходящие внутри участника процесса поставки.

Рассматривая данную модель взаимодействия, можно определить количество входящих, исходящих и внутренних процессов для каждого участника поставки товара, которые представлены в табл. 1.

Таблица 1. Количество процессов для каждого участника (классическая модель) Table 1. Number of processes for each participant (classic model)

\begin{tabular}{|l|c|c|c|c|}
\hline $\begin{array}{c}\text { Категории } \\
\text { участников }\end{array}$ & Исходящие & Входящие & Внутренние & $\begin{array}{c}\text { Всего } \\
\text { процессов }\end{array}$ \\
\hline Поставщики & 4 & 4 & 2 & 10 \\
\hline Транспорт & 3 & 3 & 3 & 9 \\
\hline
\end{tabular}




\begin{tabular}{|l|c|c|c|c|}
\hline Оптовая компания & 4 & 4 & 2 & 10 \\
\hline Розничная сеть & 5 & 5 & 2 & 12 \\
\hline Потребители & 3 & 3 & 0 & 6 \\
\hline Всего процессов & 19 & 19 & 9 & 47 \\
\hline
\end{tabular}

Общее количество процессов в рамках данной модели взаимодействия - 47. (рис. 2).

Для наглядного представления данные о количестве процессов сведены в график

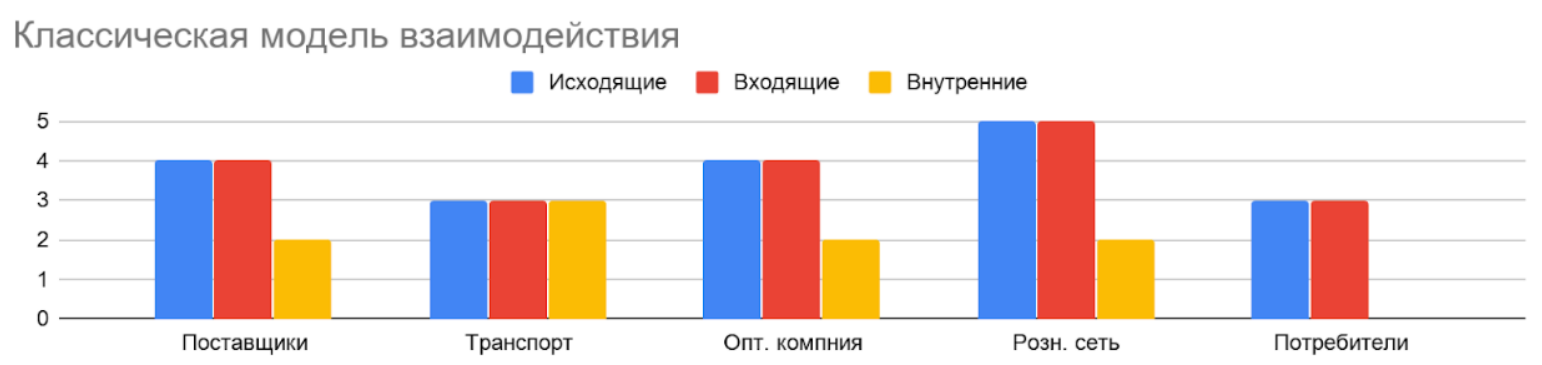

Рисунок 2. Количество процессов для каждого участника (классическая модель)

Figure 2. Number of processes for each participant (classic model)

Анализируя приведенные данные, можно сформулировать ряд положений. В частности наибольшее количество действий мы наблюдаем у таких участников, как поставщики, оптовые компании, розничные сети, что объясняется числом процессов, осуществляемых участниками поставки товара в данной модели. Так, категория «Поставщик» задействована в 4 исходящих, 4 входящих и 2 внутренних процессах; из них 6 - в рамках информирования о товаре (доведение информации о товаре до оптовых компаний, розничных сетей и потребителей и получение от них обратной связи). Кроме того, он также осуществляет упаковку товара, его складирование и учет, выступает начальным звеном физического перемещения и отслеживает финансовые расчеты. Таким образом, поставщик вовлечен в 10 процессов по поставке товара.

Оптовые и розничные компании ведут 10 и 12 процессов соответственно. Каждый из участников данных категорий задействован в физическом перемещении товара, получая его от предыдущего звена и передавая дальше по пути к конечному потребителю. Кроме того, оба осуществляют складирование и учет товара, выкладку и финансовые расчеты, информирование о товаре. Однако участник из категории «Розничная сеть» дополнительно задействован в процессе информирования о товаре конечного потребителя, доводя непосредственно до него информацию о свойствах товара и получая обратную связь.

Транспортный партнер задействован во всех процессах физического перемещения товара (9 операций).

Конечный потребитель, будучи вовлеченным в 6 процессов, контактирует главным образом с розничной сетью, покупая товар, проводя финансовые расчеты и участвуя в процессе информирования о товаре. Также потребитель может взаимодействовать в рамках процесса информирования о товаре напрямую с производителем.

На основе изложенного автор выделяет достоинства и недостатки данной модели. К достоинствам можно отнести следующее:

- высокая вовлеченность поставщика товара в процесс его поставки, что может способствовать полному соответствию товара необходимым потребительским свойствам, в т. ч. касательно сроков поставки, информированию о товаре и т. д.; 
- сравнительно небольшое количество участников процесса (снижает риск задержки процесса поставки товара).

Недостатками данной модели назовем:

- большое число процессов, выполняемых поставщиком товара, что может создавать сложности в управлении поставкой;

- многократное повторение похожих процессов, таких как складирование и учет (встречается 3 раза), выкладка товара (встречается 2 раза), неизбежно приводящее к удорожанию продукции;

- сложность информационного обеспечения, выступающей негативным явлениям в случае изменения какой-либо информации о товаре.

Модель сетевого взаимодействия основана на процессах взаимного обмена ресурсами между предпринимательскими сетями, отдельными предприятиями и другими участниками рынка. Данная модель позволяет силами участников сети создавать качественно новый продукт, который не может быть создан усилиями отдельных участников. При этом каждый из них остается независимым и может продолжать деятельность, не связанную с участием в сети. Пример модели сетевого взаимодействия представлен на рис. 3 .
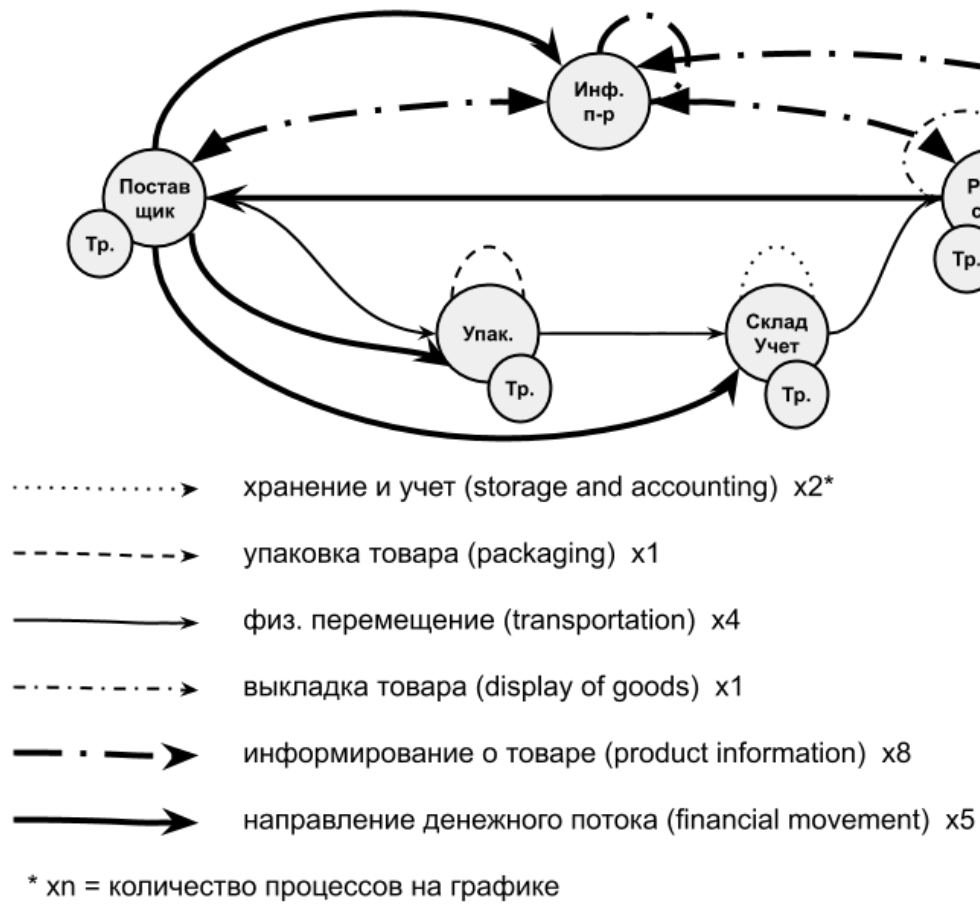

Поставщик - поставщики товара $(5 ; 2 ; 0)^{\star \star *}$

Инф. п-р - партнеры по информационной поддержке $(3 ; 4 ; 1)$ Упак. - партнеры по упаковке $(1 ; 2 ; 1)$ Склад Учет - партнеры по складированию и учету $(1 ; 2 ; 1)$ Розн. сеть - партнеры по реализации $(4 ; 4 ; 2)$

Потребитель - конечные потребители $(3 ; 3 ;-)$

Тр. - транспортные партнеры $(4 ; 4 ; 4)$ **(количество: исходящие процессы; входящие процессы; внутренние процессы)

Сумма ребер (процессов): 41 Сумма вершин (участников): 7 Количество процессов на 1 участника в среднем: 6

Рисунок 3. Схема модели сетевого взаимодействия

Figure 3. Scheme of the network interaction model

Рассматривая данную модель взаимодействия, можно определить количество входящих, исходящих и внутренних процессов для каждого участника поставки товара, которые представлены в табл. 2. 
Таблица 2. Количество процессов для каждого участника (сетевая модель) Table 2. Number of processes for each participant (network model)

\begin{tabular}{|l|c|c|c|c|}
\hline $\begin{array}{c}\text { Категории } \\
\text { участников }\end{array}$ & Исходящие & Входящие & Внутренние & $\begin{array}{c}\text { Всего } \\
\text { процессов }\end{array}$ \\
\hline Поставщики & 5 & 2 & 1 & 8 \\
\hline Упаковщик & 1 & 2 & 1 & 4 \\
\hline Склад и учет & 1 & 2 & 1 & 4 \\
\hline Транспорт & 4 & 4 & 4 & 12 \\
\hline $\begin{array}{l}\text { Информационный } \\
\text { партнер }\end{array}$ & 3 & 4 & 1 & 8 \\
\hline Розничная сеть & 4 & 4 & 2 & 6 \\
\hline Потребители & 3 & 3 & 0 & 52 \\
\hline Всего процессов & 21 & 21 & 10 & 10 \\
\hline
\end{tabular}

Общее количество процессов в рамках данной модели взаимодействия - 52 .

Для наглядности данные о количестве процессов представлены графиком (рис. 4).

\section{Модель КСВ}

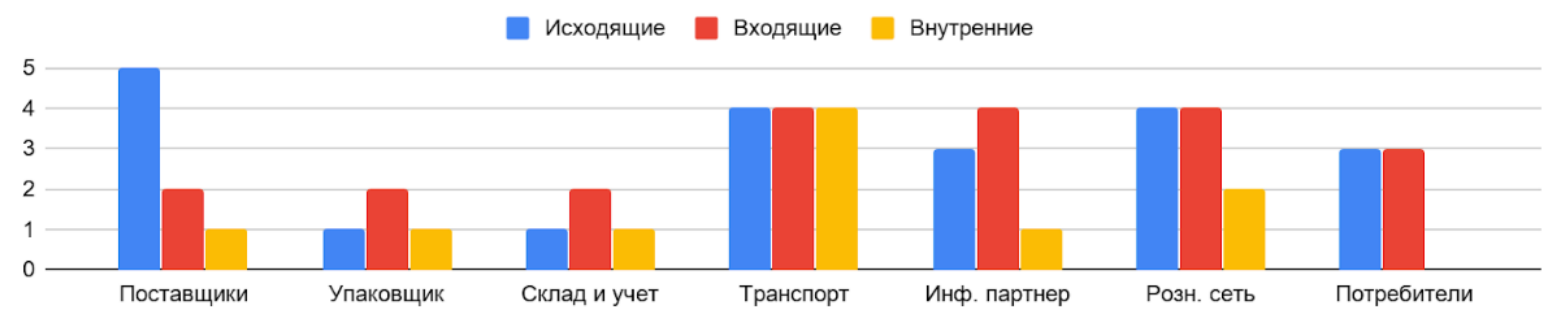

Рисунок 4. Количество процессов для каждого участника (сетевая модель)

Figure 4. Number of processes for each participant (network model)

В этой модели наибольшее количество процессов (12) мы наблюдаем у участника «Транспорт», задействованного во всех операциях, связанных с физическим движением товара. Общее число операций розничных сетей сократилось с 12 до 10. Это объясняется сокращением числа процессов информирования о товаре.

Категория участников «Поставщик» также сократила количество осуществляемых операций (с 10 до 8), при этом внутренние операции по упаковке, складированию и учету были переданы предприятиям-партнерам; процесс информирования о товаре также был передан партнеру. Данная категория участников в большей степени осуществляет контроль финансовых расчетов, что повлекло увеличение числа операций в данной группе.

Количество операций у потребителя товара осталось прежним.

Число вершин графа увеличилось за счет изменения количества участников процесса поставки добавлением таких партнеров, как информационный партнер, партнер по складированию и учету товара, партнер по упаковке. Каждый из них взял на себя ряд функций, что, в свою очередь, способствовало сокращению ряда процессов у других участников поставки товара.

К достоинствам данной модели автор предлагает отнести следующее:

- снижение количества процессов у поставщика что может способствовать повышению эффективности его работы за счет передачи части функций предприятиям партнерам; 
- способность категории участников «Склад и учет» выступать в роли некого распределительного центра для розничных сетей, работая с большим числом поставщиков и розничных сетей, владея информацией как о спросе на товар, так и о его предложении;

- повышение специализации отдельных предприятий, положительно сказывающееся на качестве конечного продукта;

- развитие подобных отношений между предприятиями, что может способствовать развитию инфраструктуры в целом.

- независимость и добровольность участия в сети;

- доступ к дополнительным ресурсам;

Недостатками данной модели назовем

- увеличение количества участников процесса поставки, требующее достаточно развитой инфраструктуры, в том числе транспортной, что может негативно отражаться на процессе поставки в случае ее отсутствия;

- увеличение количества участников процесса как риск задержки поставки товара.

Таким образом представленная модель взаимодействия имеет свои преимущества и недостатки. Она эффективна на многих предприятиях, исследуемых в рамках данной работы. В современной практике бизнеса данную модель применяют не только при поставке товара или услуг, но и в производстве, переработке и других процессах рыночного взаимодействия.

Далее рассмотрим платформенную модель взаимодействия.

Благодаря тому, что цифровые технологии становятся доступны широким массам в силу использования электронных устройств в повседневной жизни большей частью населения, создаются условия для эффективной деятельности такой бизнесмодели, как платформенное взаимодействие.

Этим термином автор обозначает взаимодействие участников рынка между собой на основе использования интернет-платформ, предлагающих различные услуги для участников такого взаимодействия.

Примерами могут служить сервисы по заказу такси, основанные на интернетплатформе, онлайн-маркетплейсы и т. д.

Модель взаимодействия участников в процессе поставки товара в платформенной модели показана на рис. 5.

Рассматривая данную модель взаимодействия, можно определить количество входящих, исходящих и внутренних процессов для каждого участника поставки товара, которые представлены в табл. 3.

Таблица 3. Количество процессов для каждого участника (платформенная модель)

Table 3. Number of processes for each participant (network model)

\begin{tabular}{|l|c|c|c|c|}
\hline $\begin{array}{c}\text { Категории } \\
\text { участников }\end{array}$ & Исходящие & Входящие & Внутренние & $\begin{array}{c}\text { Всего } \\
\text { процессов }\end{array}$ \\
\hline Поставщики & 3 & 3 & 1 & 7 \\
\hline Транспорт & 2 & 2 & 2 & 6 \\
\hline Платформа & 4 & 4 & 3 & 11 \\
\hline Потребители & 3 & 3 & 0 & 6 \\
\hline Всего процессов & 12 & 12 & 6 & 30 \\
\hline
\end{tabular}

Общее количество процессов в данной модели - 30. Представим их графиком (рис. 6). 


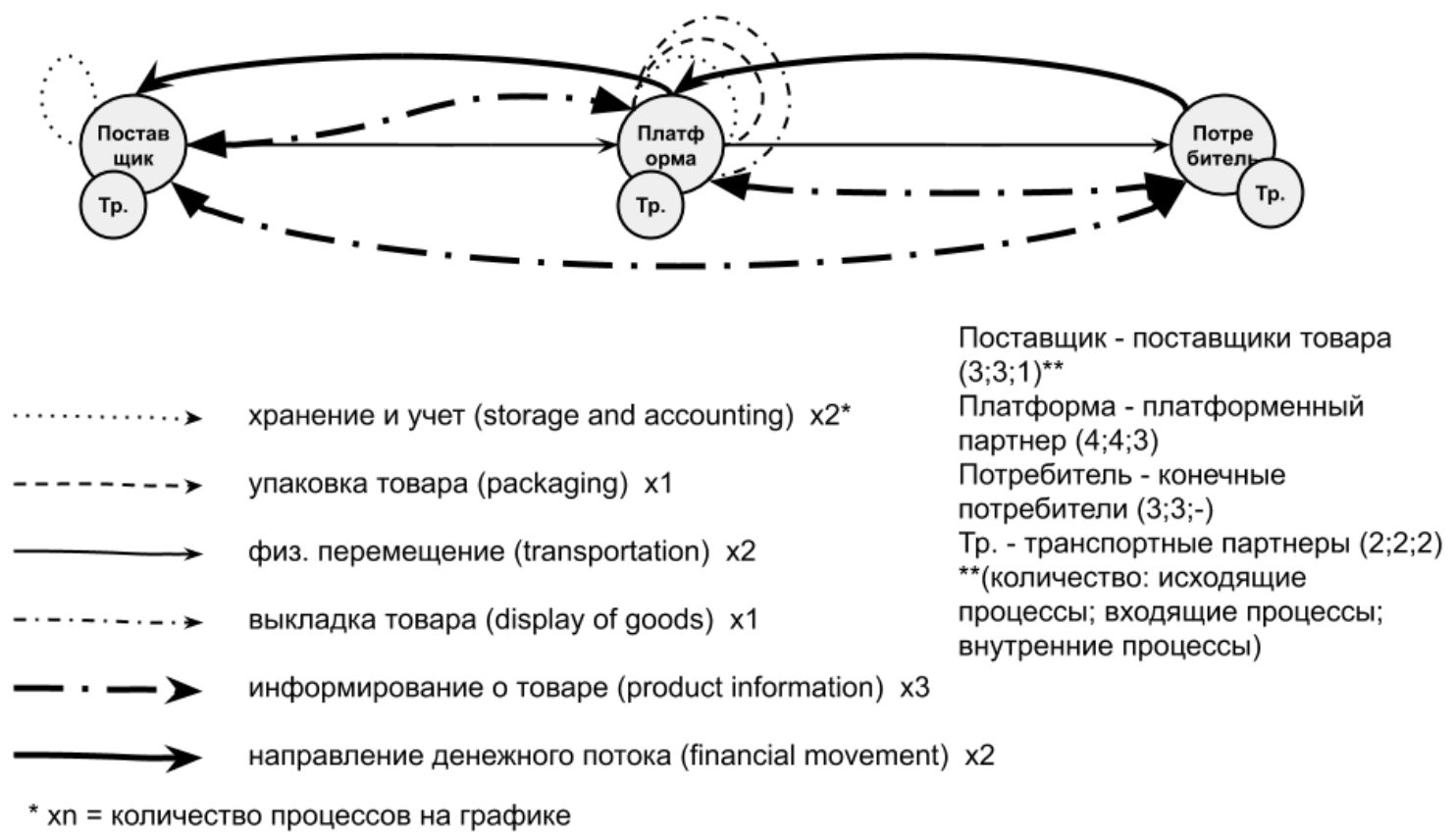

Сумма ребер (процессов): 30

Сумма вершин (участников): 4 Количество процессов на 1 участника в среднем: 7,5

Рисунок 5. Схема платформенной модели взаимодействия

Figure 5. Scheme of the platform interaction model

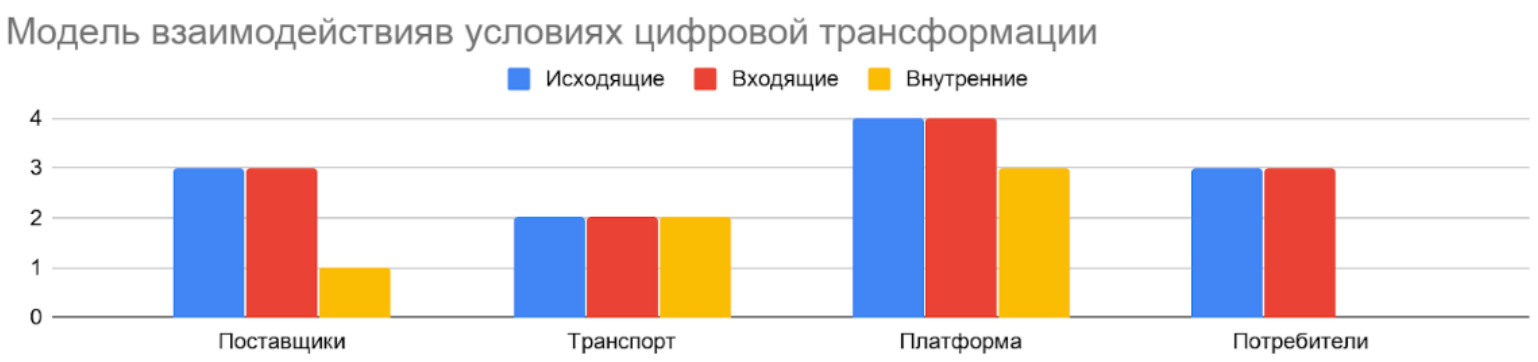

Рисунок 6. Количество процессов для каждого участника (платформенная модель) Figure 6. Number of processes for each participant (platform model)

Здесь необходимо отметить сокращение числа участников процесса. Данное положение является следствием того, что большое количество процессов взяла на себя категория участников «Платформа» (задействована в 11 процессах поставки товара: 4 исходящих, 4 входящих и 3 внутренних).

Категория участников «Поставщик» сократила количество осуществляемых операций до 7.

Транспортные партнеры также стали задействованы меньше, чем в предыдущих двух моделях, осуществляя только 6 процессов.

Количество операций у потребителя товара осталось прежним.

Граф существенно уменьшился как с позиции сокращения вершин (участников), так и с позиции числа ребер (процессов) вследствие того, что платформа позволяет осуществлять ряд процессов между поставщиком и потребителем напрямую.

К достоинствам данной модели предлагаем отнести следующее:

- сокращение посредников между поставщиком товара и потребителем; 
- снижение количества операций в процессе поставки, что может привести к снижению стоимости товара для потребителя;

- информационный обмен между участниками сети напрямую, что способствует улучшению услуг в процессе поставки;

- небольшое количество процессов физического перемещения товара, требующее более простого контроля над его передвижением;

Недостатками данной модели послужили:

- отсутствие возможности потребителя перед покупкой оценить потребительские качества товара индивидуально для собственных предпочтений (в свою очередь платформы создают условия для оценки товара теми потребителями, которые его уже приобретали, в связи с чем новый покупатель может получить некую информацию о товаре с точки зрения потребительских качеств до покупки);

- ожидание доставки товара;

- возможность столкновения с недобросовестным поставщиком;

- практика применения методов недобросовестной конкуренции путем создания заведомо отрицательных отзывов о товаре и/или услуге.

С целью сравнения моделей между собой рассмотрим количество процессов, в которых задействованы участники поставки (табл. 4).

Таблица 4. Сравнение рассмотренных моделей взаимодействия

Table 4. Comparison of the considered models of interaction

\begin{tabular}{|l|c|c|c|}
\hline \multicolumn{1}{|c|}{ Процессы } & $\begin{array}{c}\text { Классическая } \\
\text { модель }\end{array}$ & Модель КСВ & $\begin{array}{c}\text { Платформенная } \\
\text { модель }\end{array}$ \\
\hline Хранение и учет & 3 & 2 & 2 \\
\hline Упаковка & 1 & 1 & 1 \\
\hline $\begin{array}{l}\text { Физическое } \\
\text { перемещение }\end{array}$ & 3 & 4 & 2 \\
\hline Выкладка & 2 & 1 & 1 \\
\hline Информирование & 10 & 8 & 6 \\
\hline $\begin{array}{l}\text { Финансовые } \\
\text { расчеты }\end{array}$ & 3 & 5 & 2 \\
\hline
\end{tabular}

Для визуального представления предлагаем график на рис. 7.

\section{Сравнение моделей взаимодействия}

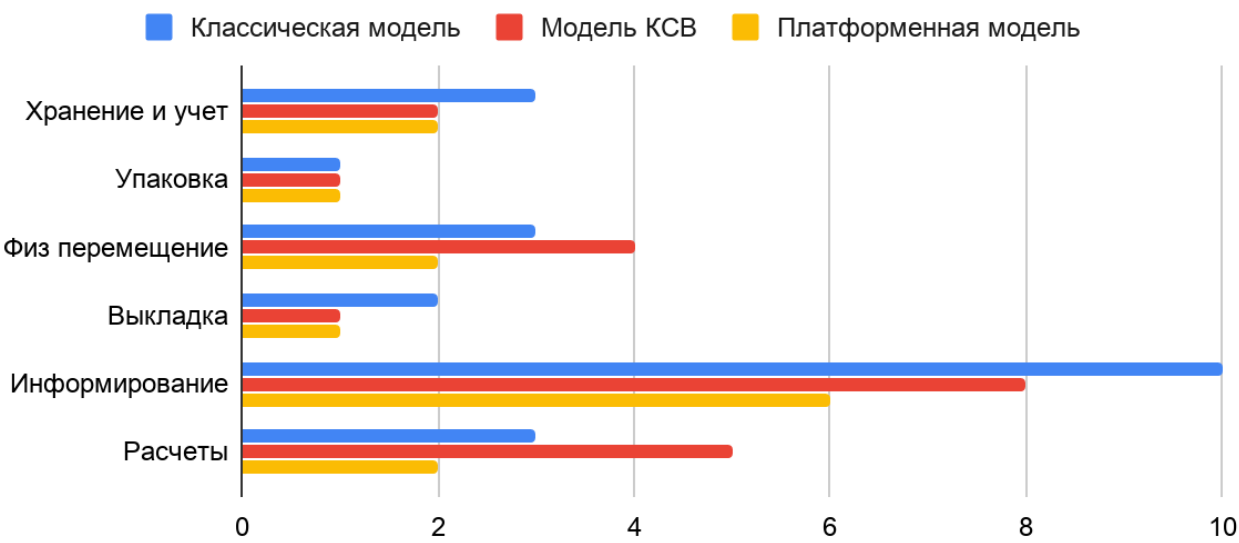

Рисунок 7. Сравнение рассмотренных моделей взаимодействия

Figure 7. Comparison of the considered interaction models 
Таким образом, процесс поставки товара на основе платформенного взаимодействия предстает как наиболее эффективная модель. Так, наблюдается снижение количества процессов во всех операциях, кроме упаковки. Число участников поставки также уменьшено. Исходя из всего сказанного, автор сформулировал ряд выводов.

1. Использование платформенной модели в современных условиях развития цифровых технологий позволяет снижать себестоимость продукции за счет упрощения процесса поставки товара от поставщика до потребителя.

2. Данная модель достаточно проста для входа новых участников рынка, что способствует развитию конкуренции.

3. Использование модели кооперационно-сетевого взаимодействия, с одной стороны, позволяет предприятиям получить доступ к новым ресурсам, что может способствовать развитию этих предприятий, с другой - предприятия предоставляют доступ к своим ресурсам другим участникам, способствуя развитию всей сети.

Полученные результаты. В ходе исследования установлено, что метод графов может быть использован в моделировании взаимодействия предприятий с внешней средой как при создании бизнеса, так и на этапе его функционирования.

Выводы и дискуссионные вопросы. Безусловно, список положений, выводов, достоинств и недостатков моделей, рассмотренный автором, не является исчерпывающим.

Необходимо дальнейшее исследование и разработка данного метода анализа. Однако уже сейчас очевидно, что его использование может помочь предприятиям провести анализ взаимодействия с внешней средой, оценить, какие процессы требуют большего внимания. Вместе с тем автор понимает, что каждое предприятие уникально и стоимость каждого процесса может существенно отличаться от одного предприятия к другому.

Исследование выполнено при финансовой поддержке РФФИ, Правительства Красноярского края и Красноярского краевого фонда науки в рамках научного проекта № 20-410-242916 р_мк_Красноярск

\section{Библиографический список}

1. Рубцова, Н. В. Методические аспекты оценки эффективности сетевого взаимодействия в сфере туристско-рекреационных услуг региона / Н. В. Рубцова // Вестник Волгоградского государственного университета. Экономика. -2020 . - Т. 22, № 1. - C. 68-82. - DOI: 10.15688/ek.jvolsu.2020.1.7

2. Кооперационно-сетевые взаимодействия как ресурс самоорганизации и достижения качественных результатов / В.В. Куимов, Ю.Ю. Суслова, Е.В. Щербенко, и др. - Москва, 2019. Сер. Научная мысль (СФУ).

3. Буркатовская, Ю.Б. Теория графов. Часть 1: учебное пособие / Ю.Б.Буркатовская; Томский политехнический университет. - Томск: Изд-во Томского политехнического университета, 2014. - 200 с

4. Гармидер, А. А. Подход к оценке синергетического эффекта от кластеризации туристической сферы региона / А. А. Гармидер // Теория и практика общественного развития. - 2015. - № 14. - С. 54-56.

5. Кузнецов, Б. Л. Эффективность корпоративного развития / Б. Л. Кузнецов // Экономическая синергетика: Ответы на вызовы и угрозы XXI века : сб. науч. тр. Набережные Челны : Изд-во Кам. гос. инж.-экон. акад., 2005. - С. 119-126. 
6. Левченко, Т. А. Кластерные структуры: основные характеристики и генерируемый эффект / Т. А. Левченко, Е. В. Тунгусова // Фундаментальные исследования. - 2017. - № 3. - С. 144-148.

7. Мингалеева, Э. К. Институционально-синергетический механизм управления трансакционными издержками вертикально-интегрированной корпорации : автореф. дис. ... канд. экон. наук / Мингалеева Эльмира Камиловна. - Набережные Челны : ИНЭКА, 2009. - 28 с.

8. Мусаев, Л. А. Оценка синергетического эффекта экономических систем / Л. А. Мусаев // Вестник ЮРГТУ (НПИ). - 2011. - № 3. - С. 132-137.

9. Никулина, Т. А. Методика оценки синергетического эффекта функционирования корпоративных розничных торговых сетей / Т. А. Никулина, И. М. Романова, Ю. Д. Шмидт // Экономика и предпринимательство. - 2015. - № 3. - С. 939945.

10. Замятин А. П. 3-269 Графы и сети: учеб. пособие. - Екатеринбург: Изд-во Урал, ун-та, 2004. -- 160 с. - ISBN 5-7996-0308-7.

\section{References}

1. Rubtsova, N.V. (2020). Methodical Aspects of Evaluating the Effectiveness of Network Interaction in the Field of Tourist and Recreational Services in a Region. Journal of Volgograd State University. Economics. 22(1). 68-82. (in Russian). DOI: https://doi.org/10.15688/ek.jvolsu.2020.1.7

2. Kuimov, V.V., Suslova, Yu.Yu., Shcherbenko E.V. et al. (2019). Cooperation and network interactions as a resource for self-organization and achievement of high-quality results. Moscow.

3. Burkatovskaya, Yu.B. (2014). Graph theory. Tomsk : Tomsk./ polit. univer.

4. Garmider, A.A. (2015). Approach to assessing the synergistic effect of clustering tourism in the region. Theory and Practice of Social Development. 14. 54-56.

5. Kuznetsov, B.L. (2005). Corporate Development Efficiency. Economic Synergy: Answers to the Challenges and Threats of the 21st Century. Collection of Scientific Works. Naberezhnye Chelny. 119-126.

6. Levchenko, T.A., Tungusova, E.V. (2017). Cluster Structures: Main Characteristics and Generated Effect. Fundamental research. 3. 144-148.

7. Mingaleeva, E.K. (2009). Institutional Synergistic Mechanism for Managing the Transaction Costs of a Vertically Integrated Corporation. Cand. econ. sci. abs. diss. Naberezhnye Chelny. 28 p.

8. Musaev, L.A. (2011). Evaluation of the Synergistic Effect of Economic Systems. The Bulletin of the SouthRussian State Technical University (NPI). 3. 132-137.

9. Nikulina, T.A., Romanova, I.M., Shmidt, Yu.D. (2015). Methodology for Assessing the Synergistic Effect of Corporate Retail Chains. Journal of Economy and Entrepreneurship. 2015. 3. 939--945.

10. Zamyatin, A. P. (2004). 3-269 Graphs and networks. Yekaterinburg : Ural, un-t. 160 p. ISBN 5-7996-0308-7. 